\title{
Topological Basis of Flat Electroencephalography's State Space
}

\author{
Tan Lit Ken ${ }^{1, *}$, Tahir bin $\mathrm{Ahmad}^{2}$, Lee Kee Quen ${ }^{3}$, Gan Yee Siang ${ }^{4}$, Tey Wah Yen ${ }^{5}$ and Wong Wai Kit ${ }^{6}$ \\ ${ }^{1,3}$ Department of Mechanical Precision Engineering, Malaysia-Japan International Institute of Technology \\ Universiti Teknologi Malaysia, UTM KL, Jalan Sultan Yahya Petra, 54100 Kuala Lumpur, Malaysia \\ ${ }^{2}$ Department of Mathematical Sciences and Centre of for Sustainable Nanomaterials, \\ Universiti Teknologi Malaysia, 81310 Skudai, Johor Darul Takzim, Malaysia \\ ${ }^{4}$ Department of Mathematics, Xiamen University Malaysia, \\ 43900 Sepang, Selangor Darul Ehsan, Malaysia \\ ${ }^{5}$ Faculty of Engineering, Technology \& Built Environment, UCSI University, \\ North Wing Campus, 56000 Cheras, Kuala Lumpur, Malaysia \\ ${ }^{6}$ Department of Anesthesiology, Hospital Selayang, Lebuh Raya Selayang-Kepong, \\ 68100 Batu Caves, Selangor Darul Ehsan, Malaysia \\ *Corresponding author's email: tlken [AT] utm.my
}

\begin{abstract}
Neuroinverse problem are often associated with complex neuronal activity. It involves locating problematic neuron which usually a highly challenging task. While epileptic foci localization is possible with the aid of EEG signals, it relies greatly on the ability to extract hidden information or pattern within EEG signals. Flat EEG being an enhancement of EEG is a way of viewing electroencephalograph on the real plane. In the perspective of dynamical systems, the equivalency of epileptic seizure and Flat EEG is important since the underlying structure of Flat EEG is dynamic. In this paper, a basis for the topological space of Flat EEG is presented. It is expected to facilitate in the constructions of dynamic transformation from epileptic seizure to Flat EEG. It provides a better understanding on Flat EEG itself.
\end{abstract}

Keywords_- Epilepsy, Flat EEG, Topology, Dynamical Systems.

\section{INTRODUCTION}

Epilepsy is one of the most common brain disorders in the world. This disorder has no age, gender, racial, social class, nationality nor geographic boundaries. In other words, it can happen to anyone in the world. According to the Commission on Epidemiology and Prognosis and International League Against Epilepsy 1993, epilepsy can be defined as an "occurrence of at least two unprovoked seizures" (Panayiotopoulos, 2010), that is, it usually occur more than once and is not prompted. This brain disorder is not infectious, that is, it is not passed down through families. In fact, people can't "catch it". Statistically, the number of people with epilepsy in the world is at least 50 millions in total which is roughly $1.5 \%$ of the world populations (Jahnecke et. al., 2006)

Epilepsy is not related to mental illness. Instead, it is a condition of the brain. It generally does not affect the learning and thinking ability. As a matter of fact, most people with epilepsy have normal mental intelligence, with some having superior cognitive abilities (Wyllie et al., 2006). However, epilepsy can limits one's independence, freedom and quality of life. For examples, a person with epilepsy has limited job options, has trouble on getting a driving license, facing social rejection, discrimination or prejudice due to other people's fears and misconceptions about his or her condition. Epilepsy is dangerous as it can lead to fatality especially when a seizure continues for a long period of time and causes brain damage. Such situation is known as epilepticus.

Two of the methods of controlling or stopping seizures are by taking anticonvulsant medications and undergo surgery. The former method is challenging in terms of finding the exact combinations of medications and dosages. Besides, this method is not promising because some seizures can still failed to be controlled in spite with the best available medications. Moreover, anticonvulsant medications carry undesired side effect (Sharon et al., 2004; Stefan and Feuerstein, 2007). The effects are based on the type of anticonvulsant medications one consumed. Frequently, it adversely affects the cognitive ability of patients (Bennett, 1992). Consequently, some patients choose to undergo 
epilepsy surgery. As a matter of fact, neurologists often suggest surgery as the best solution. The target of this method is to remove problematic epileptogenic tissue while sparing essential brain areas to avoid neurologic deficits. Therefore, determining the exact location of problematic cells i.e., epileptic foci is crucial.

Electroencephalography is the process of obtaining electrical potentials produced by neurons inside the brain. An instrument called electroencephalograph (Figure 1) is used. With this instrument, the summed electrical activities due to ionic current flows between neurons during the transmission of information underneath the electrodes can be recorded noninvasively from the scalp. This process last 20 up to 40 minutes. Electroencephalography is very important apparatus in neurosurgery as it is able to detect abnormal patterns of electrical activity inside the brain. The advantage of this technology, other than harmless in diagnosing epileptic seizure is also one of the most economic methods (Kiymik et al., 2005).

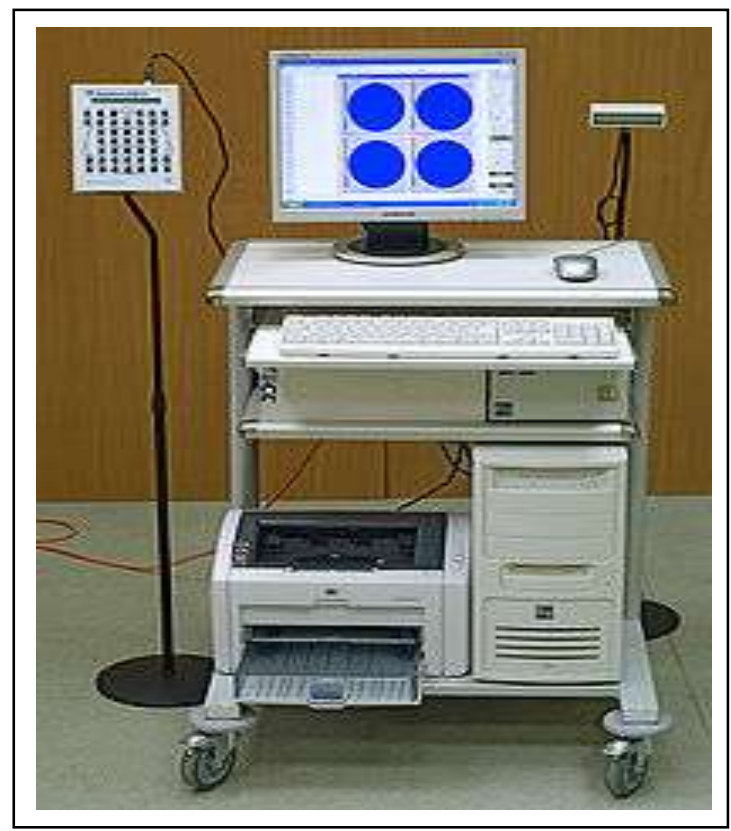

Figure 1. EEG signal (www.wikipedia.com)

In the Introduction section, present clearly and briefly the problem investigated, with relevant references. The main results should be enunciated.

\section{LITERATURE REVIEW}

All Various research using different concepts and techniques to identify epileptic foci has been established in the interest of creating better life for epileptic patients. For examples, multimodality approach (Desco et al., 2001), using large-area magnetometer and functional brain anatomy (Tiihonen et al., 2004), examining correlations among electrodes captured by linear, nonlinear and multi linear data analysis technique (Evim, A. et al., 2006), 3-D source localization of epileptic foci by integrating EEG and MRI (Natasa, M. et al., 2005) and even approaches that are based on statistical tools such as Bayesian method (Toni et al., 2005) and maximum likelihood estimation approach by Jan et al. (2004). Each of these methods has their own advantages and weaknesses.

Fuzzy Topographic Topological Mapping (FTTM) is a fuzzy and topological based model for solving neuromagnetic inverse problem (Figure 2). It consists of four components i.e., Magnetic Contour Plane (MC), Base Magnetic Plane (BM), Fuzzy Magnetic Field (FM) and Topographic Magnetic Field (TM), each of these components are homeomorphic to one another (Liau, 2001). The model is capable of portraying current sources topographically in three dimensions space. The advantage of this method is that, it does not need priori information and it is not time consuming (Tahir et al., 2005). 


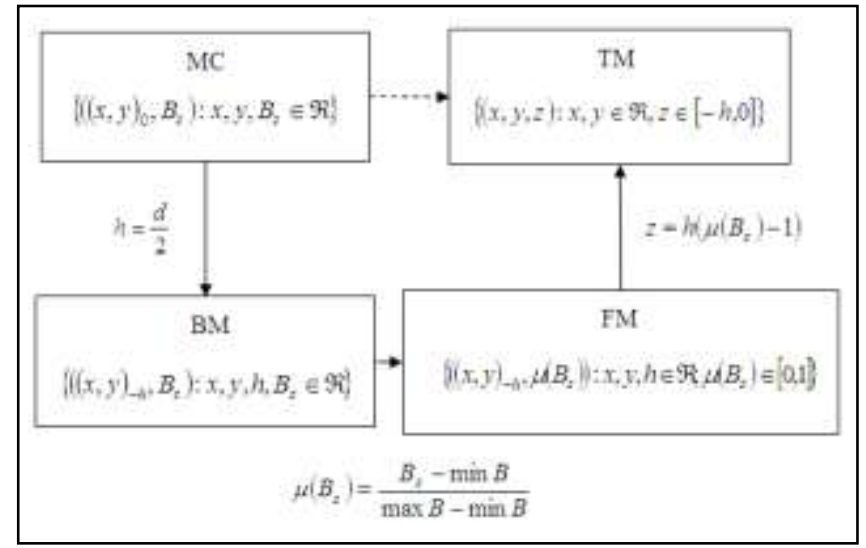

Figure 2. FTTM

A novel method called Flat electroencephalography (Flat EEG) (Figure 3) was introduced to view EEG signals on the first component of Fuzzy Topographic Topological Mapping (FTTM). It enables EEG signals be portrayed in 3 dimension space (Fauziah, 2008). This method consists of a flattening procedure (a stereographic projection) which serves as the transformation from EEG to MC (Figure 4). The main scientific value of this method lies in its ability to preserve the orientation and magnitude of EEG signals to MC, allowing it to be compressed and analyzed. Hence, performing analysis on EEG signals would allow researchers to reveal interesting pattern contained within the signals.

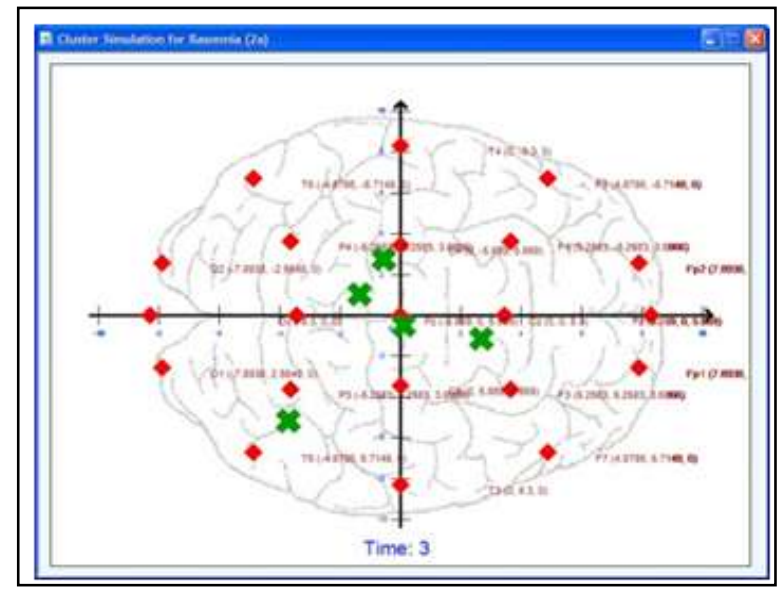

Figure 3. A Flat EEG at $t=3$

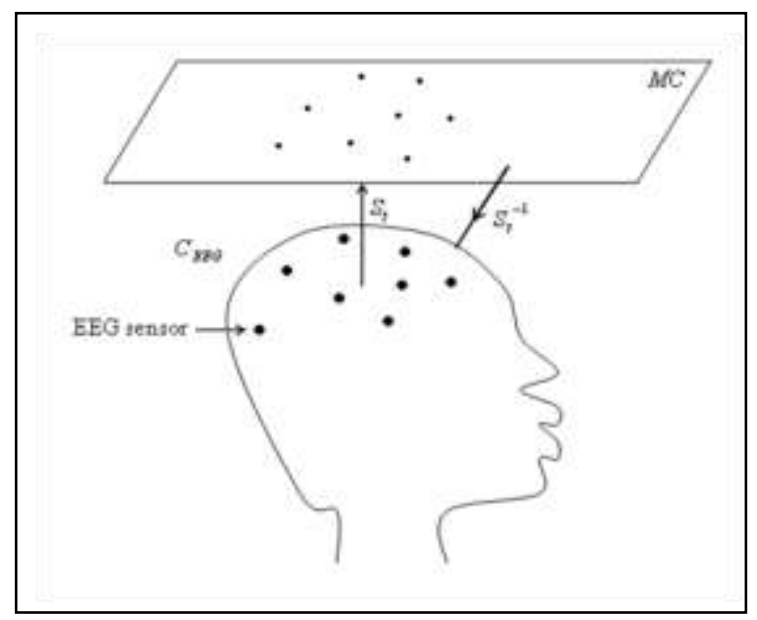

Figure 4. Stereographic projection 
A dynamical system is a system whose temporal evolution from some initial state is dictated by a set of rules (Eduard et al., 1999). Another way to understand this is, it consists of a set of variables that describe its state and a law that describe the evolution of the state variables with time, i.e., how the state of the system in the next moment of time depends on the input and its state in the previous moment of time (Eugene, 2007). This notion was adopted by Fauziah (2008) and Tan et. al., (2015) respectively, to model epileptic seizure and Flat EEG as dynamical system (Figure 5). Basically, they viewed an event of epileptic seizure and an event of Flat EEG as a trajectory in the Euclidean space $\mathfrak{R}^{m}$ and $\mathfrak{R}^{n}$ respectively.

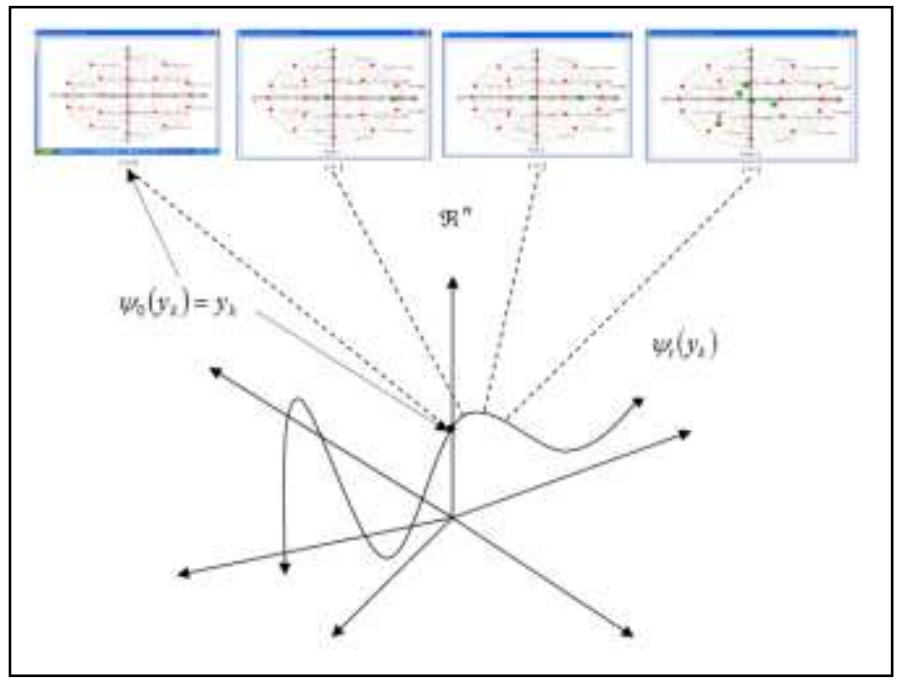

Figure 5. Dynamical system of Flat EEG

Throughout the years, various mathematical tools have been applied on Flat EEG to extract hidden information that is hardly noticeable by traditional visual inspection. Basically, Flat EEG is a platform which enables EEG signals be studied on the Cartesian plane mathematically, hence allowing the extraction of "hidden" information within EEG signals that cannot be obtained by traditional visual inspection. Furthermore, characteristics and properties obtained from this platform can be used to describe epileptic seizure since epileptic seizure and Flat EEG are topologically conjugated (Tahir and Tan; 2010, 2011) (Figure 6).

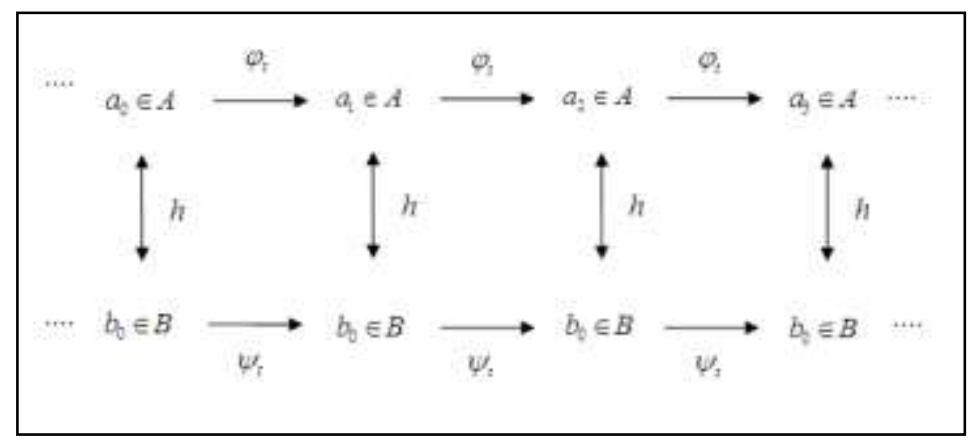

Figure 6. Pictorial representation of topological conjugacy.

There have been numerous researches utilizing various mathematical tools to visualize and extract "hidden" information within EEG signals via Flat EEG on a particular frame (or time) with promising outcome. For instance, implementation of Fuzzy C-Means (FCM) on Flat EEG enables one to compute the number of cluster centers along with its locations. It leads to the possibility of brainstorm tracking (Fauziah, 2008).

Apart from that, the algebraic study on Flat EEG demonstrates not only the possibility of transforming Flat EEG from one mathematical structure to another i.e., from topological to algebraic but also shows that Flat EEG can be decomposed 
into semigroup of upper triangular matrices under matrix multiplication and hence revealing that EEG patterns recorded during epileptic seizure is not chaotic after all (Faisal and Tahir, 2010).

Furthermore, study on the dynamic structure of Flat EEG, in particular, its structural stability from topological viewpoint, proves that Flat EEG in the presence of artifacts could still offer significant descriptions of electrical activities in the brain during seizure attack (Tan and Tahir, 2014) (see Figure 6).

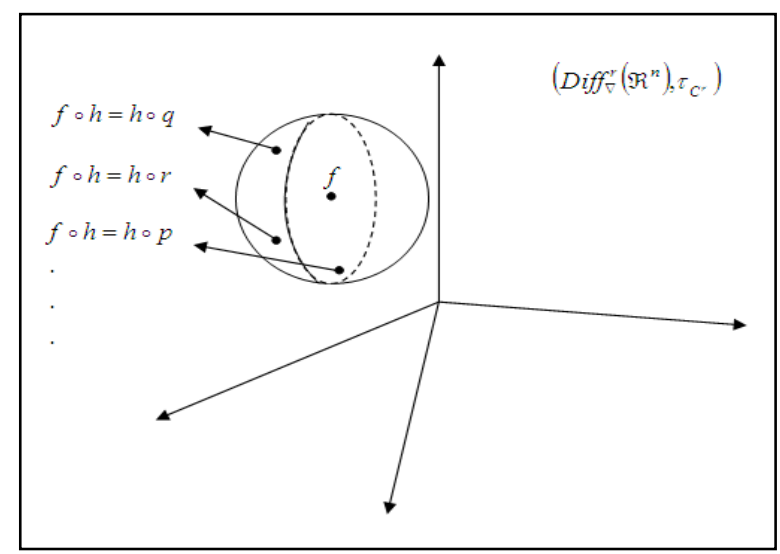

Figure 6. A structurally stable diffeomorphism $f$ in $\left(\operatorname{Diff}_{\nabla}^{r}\left(\Re^{n}\right), \tau_{C^{r}}\right)$

Several topological properties on Flat EEG's state space were established in (Tan et. al., 2015) which serve as the groundwork for dynamic topological transformation from epileptic seizure to Flat EEG i.e., in justifying Flat EEG as an alternative platform to study epileptic seizure. In this paper, a topological basis of Flat EEG's state space is presented. This finding is expected to facilitate in the construction of dynamic transformation from epileptic seizure to Flat EEG.

\section{MATERIALS AND METHODS}

Firstly, consider the flow of Flat EEG modeled in (Tahir and Tan, 2010). The function

$\psi_{t}(y)$ such that $\psi: \mathfrak{R} \times Y \rightarrow Y$ with the following two properties are fulfilled:

i. $\quad \psi_{0}(y)=y \quad \forall y \in Y=\mathfrak{R}^{n}$, and

ii. for all $t$ and $s \in \mathfrak{R}$

$$
\psi_{t} \circ \psi_{s}=\psi_{t+s}
$$

Here, for any $y_{k} \in Y=\mathfrak{R}^{n}, \psi_{t}(y)$ is generally defined as $\psi_{i}\left(y_{k}\right)=y_{i}$ i.e., the state of the system which initiates from $y_{k}$ at time $i$ is $y_{i}$

Basically, for each $y_{k} \in Y=\mathfrak{R}^{n}$, the flow of Flat EEG defines a trajectory that represents an event of Flat EEG.

Let a trajectory be denoted as $O_{\psi_{t}\left(y_{k}\right)}$, then $\left(O_{\psi_{t}\left(y_{k}\right)}, \tau_{O_{\psi_{t}}\left(y_{k}\right)}\right)$ is a topological space where $\tau_{O_{\psi_{t}\left(y_{k}\right)}}$ is the order topology induced naturally from the order structure contained within the trajectory (Tan and Tahir, 2010).

Further, let the state space be denoted as $Y=\coprod_{k \in A} O_{\psi_{t}\left(y_{k}\right)}$ i.e., the disjoint union of all the non-intersecting trajectories, then $\left(Y, \tau_{Y}\right)$ is a topological space where $\tau_{Y}$ is the disjoint union topology which can be defined as

$$
\tau_{Y}=\left\{\beta_{1} \cup \beta_{2} \cup \ldots \cup \beta_{a}: \beta_{1} \in \tau_{O_{\psi_{t}\left(y_{1}\right)}}, \beta_{2} \in \tau_{O_{\psi_{t}\left(y_{2}\right)}}, \ldots, \beta_{a} \in \tau_{O_{\psi_{t}\left(y_{a}\right)}}\right\}
$$


where,

$$
\begin{aligned}
& \tau_{O_{\psi_{t}\left(y_{1}\right)}}=\left\{\beta_{1}: \beta_{1}=\bigcup_{i=1}\left(\bigcap_{j=1}^{n} s_{1 j}\right)_{i}\right\} \\
& \tau_{O_{\psi_{t}\left(y_{2}\right)}}=\left\{\beta_{2}: \beta_{2}=\bigcup_{i=1}\left(\bigcap_{j=1}^{n} s_{2 j}\right)_{i}\right\} \\
& \cdot \\
& \cdot \\
& \tau_{O_{\psi_{t}\left(y_{a}\right)}}=\left\{\beta_{a}: \beta_{a}=\bigcup_{i=1}\left(\bigcap_{j=1}^{n} s_{a j}\right)_{i}\right\}
\end{aligned}
$$

It is not difficult to realize that equation (1) is generated by a basis. Formally, the basis can be written as

$$
B_{Y}=\left\{\gamma_{1} \cup \gamma_{2} \cup \ldots \cup \gamma_{a}: \gamma_{1} \in B_{O_{\psi_{t}\left(y_{1}\right)}}, \gamma_{2} \in B_{O_{\psi_{t}\left(y_{2}\right)}}, \ldots, \gamma_{a} \in B_{O_{\psi_{t}\left(y_{a}\right)}}\right\}
$$

Theorem 1: If $\tau_{Y}=\left\{\beta_{1} \cup \beta_{2} \cup \ldots \cup \beta_{a}: \beta_{1} \in \tau_{O_{\psi_{t}\left(y_{1}\right)}}, \beta_{2} \in \tau_{O_{\psi_{t}\left(y_{2}\right)}}, \ldots, \beta_{a} \in \tau_{O_{\psi_{t}\left(y_{a}\right)}}\right\}$ is the disjoint union topology on $Y=\coprod_{k \in A} O_{\psi_{t}\left(y_{k}\right)}$, then it is generated by the basis

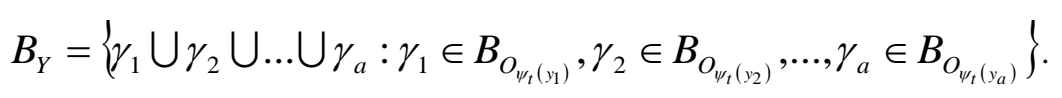

\section{Proof:}

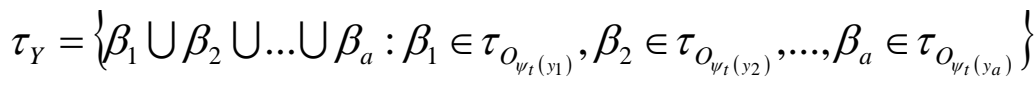

From equation $1, \beta_{k}=\bigcup_{i=1}\left(\bigcap_{j=1}^{n} s_{k j}\right)_{i}$ for all $1 \leq k \leq a$,

$$
\tau_{Y}=\left\{\left[\begin{array}{l}
\left.\left[\bigcup_{i=1}\left(\bigcap_{j=1}^{n} s_{1 j}\right)_{i}\right] \cup\left[\bigcup_{i=1}\left(\bigcap_{j=1}^{n} s_{2 j}\right)_{i}\right] \cup \ldots \cup\left[\bigcup_{i=1}\left(\bigcap_{j=1}^{n} s_{a j}\right)_{i}\right]\right\} \\
: s_{1 j} \in S_{O_{\psi_{t}\left(y_{1}\right)}}, s_{2 j} \in S_{O_{\psi_{t}\left(y_{2}\right)}}, \ldots, s_{a j} \in S_{O_{\psi_{t}\left(y_{a}\right)}}
\end{array}\right\}\right.
$$

Expanding $\left[\bigcup_{i=1}\left(\bigcap_{j=1}^{n} s_{k j}\right)_{i}\right]$ for all $1 \leq k \leq a$ 


$$
\tau_{Y}=\left\{\begin{array}{l}
\left.\qquad\left[\bigcap_{j=1}^{n} s_{1 j}\right)_{1} \cup\left(\bigcap_{j=1}^{n} s_{1 j}\right)_{2} \cup\left(\bigcap_{j=1}^{n} s_{1 j}\right)_{3} \cup \ldots\right] \\
\left.\cup\left[\bigcap_{j=1}^{n} s_{2 j}\right)_{1} \cup\left(\bigcap_{j=1}^{n} s_{2 j}\right)_{2} \cup\left(\bigcap_{j=1}^{n} s_{2 j}\right)_{3} \cup \ldots\right] \\
\cup \ldots \cup\left[\left(\bigcap_{j=1}^{n} s_{a j}\right)_{1} \cup\left(\bigcap_{j=1}^{n} s_{a j}\right)_{2} \cup\left(\bigcap_{j=1}^{n} s_{a j}\right)_{3} \cup \ldots\right.
\end{array}\right\}
$$

Rearranging the terms,

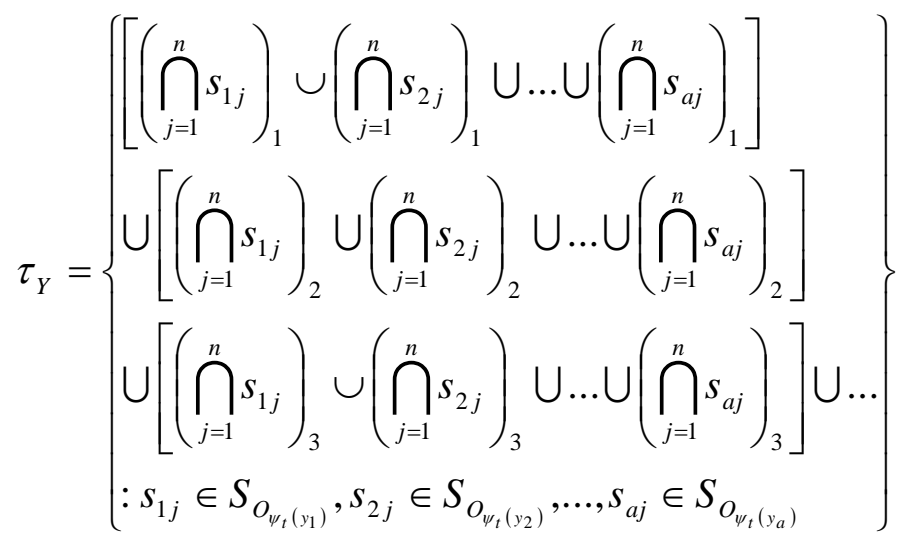

Notice that, $\left(\bigcap_{j=1}^{n} s_{k j}\right)_{i}$ for all $1 \leq k \leq a$ and $i$ is simply the basis element form by the finite intersection of subbasis. Consequently, $\left(\bigcap_{j=1}^{n} s_{k j}\right)_{i}$ for all $1 \leq k \leq a$ and $i$ can be rewritten as $\gamma_{k i}$ where $\gamma_{k i} \in B_{O_{\psi_{t}\left(y_{k}\right)}}$. That is, $\tau_{Y}=\left\{\begin{array}{l}\left(\gamma_{11} \cup \gamma_{21} \cup \ldots \cup \gamma_{a 1}\right) \cup\left(\gamma_{12} \cup \gamma_{22} \cup \ldots \cup \gamma_{a 2}\right) \\ \cup\left(\gamma_{13} \cup \gamma_{23} \cup \ldots \cup \gamma_{a 3}\right) \cup \ldots: \gamma_{1 i} \in B_{O_{\psi_{t}\left(y_{1}\right)}}, \gamma_{2 i} \in B_{O_{\psi_{t}\left(y_{2}\right)}, \ldots, \gamma_{a i}} \in B_{O_{\psi_{t}\left(y_{a}\right)}}\end{array}\right\}$

This shows that an open set in the disjoint union topology on $Y=\coprod_{k \in A} O_{\psi_{t}\left(y_{k}\right)}$ can be expressed as a union of basis elements obtained from their respective order topologies. Therefore, the basis for the disjoint union topology on $Y=\coprod_{k \in A} O_{\psi_{t}\left(y_{k}\right)}$ can be denoted as

$B_{Y}=\left\{\gamma_{1} \cup \gamma_{2} \cup \ldots \cup \gamma_{a}: \gamma_{1} \in B_{O_{\psi_{t}\left(y_{1}\right)}}, \gamma_{2} \in B_{O_{\psi_{t}\left(y_{2}\right)}}, \ldots, \gamma_{a} \in B_{O_{\psi_{t}\left(y_{a}\right)}}\right\}$. 


\section{RESULTS}

The trajectories of Flat EEG that are modeled as dynamical system are topological spaces. Furthermore, by the disjoint union topology, the union of all these trajectories, which formed the state space of Flat EEG's dynamical system, is also a topological space. This topology is generated by a basis that can be expressed as the union of basis element obtained from their respective order topologies.

\section{DISCUSSIONS}

Flat EEG's state space is a topological space and is well-behaved. Since it is generated by a basis, study on the topological properties of Flat EEG's state space can now be performed on the basis, a smaller set which is a subset to the disjoint union topology.

\section{CONCLUSIONS}

In this paper, a basis for Flat EEG's state space is presented. This finding is essential as it is expected to facilitate in the constructions of dynamic transformation from epileptic seizure to Flat EEG, particularly, in proving the bicontinuous property of the dynamic transformation from epileptic seizure to Flat EEG. Besides, it provides a better understanding on the topological properties of epileptic seizure and Flat EEG since the exploration on topological properties is possible via the basis instead of its topology.

\section{ACKNOWLEDGEMENT}

The authors would like to thank their family members and friends, especially Sally Su Li Ying, for their continuous support and assistance. The authors would also like to express his appreciation to Universiti Teknologi Malaysia and Takasago Thermal Engineering research grant, Vot No. 4B211 for financial support. This research is also partially supported by the unversiti GUP Tier 1 grant Vot No. $15 \mathrm{H} 42$.

\section{REFERENCES}

[1] Panayiotopoulos, C. P., Atlas of Epilepsies, $1^{\text {st }}$ Edition, Springer-Verlag, London Limited. 2010; Chapter 1, pp: 5.

[2] Jahnecke, C. A. N., Schwarz, L., Sovierzoski, M. A., Azevedo, D. F. M. and Argoud, F. I. M., "C++ Video-EEG Processing System With Sights to the Epileptic Seizure Detection", World Congress on Medical Physics and Biomedical Engineering, IFMBE Proceedings, 2007; 14(8): 1052-1055.

[3] Wyllie, E., Gupta, A. and Lachhwani, D.K., The treatment of epilepsy: principles \& practice, $4^{\text {th }}$ Edition, Lippincott Williams \& Wilkins, USA. 2006; pp: 1185.

[4] Sharon, R., David, I.M. and Shlomo, Y., "Anticonvulsant efficiency, behavioral performance and cortisal levels: a comparison of carbamazepine (CBZ) and a fatty acid compound (SR-3)", Elsevier, Psychoneuroendocrinology. 2004; 29(2): 113-124.

[5] Stefan, H. and Feuerstein, T. J., "Novel Anticonvulsant Drugs", Elsevier, Pharmacology \& Therapeutics. 2007; 113(1): 165-183.

[6] Bennett, T. L., Ciritical issue in Neuropsychology: The Newropsychology of Epilepsy, $2^{\text {nd }}$ Edition, Springer. 1992 ; pp: 88 .

[7] Kiymik, M.K., Guler, I., Dizibuyuk, A. and Akin, M., "Comparison of STFT wavelet transform methods in determining epileptic seizure activity in EEG signals for real-time application", Elsevier, Computers in Biology and Medcine. 2005; 35(7): 603-616.

[8] Desco, M., Pascau, J., Pozo, M. A., Santos, A., Reig, S., Gispert, J. and Garcia, B. P., "Multimodality localization of epileptic foci", Physiology and Function from Multidimensional Images, Proceedings of SPIE, Medical Imaging. 2001; 4321(1): pp: 362-370.

[9] Tiihonen, J., Hari, R., Kjola, M., Nousiainen, U. and Vapalahti, M., "Localization of epileptic foci using large-area magnetometer and functional brain anatomy", Wiley, Annals, of Neurology. 2004; 27(3): 283-290.

[10]Evim, A., Canan, A. B., Haluk, B. and Bulent, Y., "Computational analysis of epileptic focus localization", ACTA Press Anaheim, BioMed'06 Proceedings of the $24^{\text {th }}$ IASTED international conference on Biomedical Engineering. 2006; pp 317-322.

[11] Natasa, M., Malek, D., Ilker, Y. and Prasanna, J., "3-D source Localization of Epileptic Foci Integrating EEG and MRI Data”, Springer-Verlag, Brain Topography. 2003; 16(2): 111-119.

[12] Toni, A., Aopa, N., Matti, S. H., Liro, P. J. Jouko, L., Aki, V. and Mikko, S., "Bayesian analysis of the neuromagnetic inverse problem with $\mathrm{l}^{\mathrm{p}}$-norm priors", Elsevier, Neuroimage. 2005; 26(3): 870-884. 
[13] Jan, C. D. M., Fetsje, B., Pawel, G., Cezary, A. S., Maria, I. B. and Heethaar, R. M. A., "Maximum-Likelihood Estimator For Trial-to-Trial Variations in Noisy MEG/EEG Data Sets", IEEE Transactions on Biomedical Engineering. 2004; 51(2): 2123-2128.

[14]Liau, L. Y., "Homeomorfisma Antara $S^{2}$ Dan $E^{2}$ MelaluiStrukturPermukaan Riemann Serta Deduksi Teknik Pembuktiannya Bagi Homeomorfisma Pemetaan Topologi Topografi Kabur (FTTM)", Master Thesis, UniversitiTeknologi Malaysia, Skudai. 2001.

[15] Tahir, A., Rashdi, S. A., Liau, L. Y., Fauziah, Z. and Wan Eny Zarina, W. A. R., ”Homeomorphisms of Fuzzy Topographic Topological Mapping (FTTM)", UTM, Matematika. 2005; 21(1): 35-42.

[16]Fauziah, Z., "Dynamic Profiling Of Electroencephalography Data During Seizure Using Fuzzy Information Space", UniversitiTeknologi Malaysia, PhD Thesis, Skudai. 2008.

[17]Eduard, G., Helwig, L. and Raine, W., "Visualization of Dynamical Systems. Elsevier, Future Generation Computer Systems", 1999; 15(1): 75-86.

[18]Eugene, M. I., "Dynamical System in Neuroscience: The Geometry of Excitability and Bursting", The MIT Press Cambridge, Massachusetts London England. 2007; pp: 8.

[19] Tan, L. K., Tahir, A., Mohd, S. M. and Su. K. N., "Dynamical System of Flat Electroencephalography", Science Publications, American Journal of Applied Sciences. 2015; 12(7): 495-505.

[20] Tahir, A. and Tan, L. K., "Topological Conjugacy between Seizure and Flat Electroencephalography”, Science Publications, American Journal of Applied Sciences. 2010; 7(3): 1470-1476.

[21] Tahir, A. and Tan, L. K., "Flows of Continuous-Time Dynamical Systems with No Periodic Ordbit as an Equivalence Class under Topological Conjugacy Relation”, Science Publications, Journal of Mathematics and Statistics. 2011; 7(3): 207-215.

[22]Faisal, A. M. B. and Tahir, A., "EEG Signals During Epileptic Seizure as a Semigroup of Upper Triangular Matrices", Science Publications, American Journal of Applied Sciences. 2010; 7(4): 540-544.

[23] Tan, L. K. and Tahir, A., "Structural Stability of Flat Electroencephalography", Marsland Press, Life Science Journal-Acta Zhengzhou University Overseas Edition. 2014; 11(8): 165-170. 